Research Paper

\title{
Analysis of Origin and Protein-Protein Interaction Maps Suggests a Distinct Oncogenic Role of Nuclear EGFR during Cancer Evolution
}

\author{
Ainur Sharip ${ }^{1 *}$, Diyora Abdukhakimova ${ }^{*}$, Xiao Wang 2 , Alexey Kim ${ }^{1}$, Yevgeniy Kim ${ }^{1}$, Aigul Sharip ${ }^{1}$, \\ Askarbek Orakov ${ }^{1}$, Lixia Miao ${ }^{3}$, Qinglei Sun², Yue Chen" ${ }^{4}$ Zhenbang Chen ${ }^{5}$ and Yingqiu Xie ${ }^{1 * \bowtie}$ \\ 1. Department of Biology, School of Science and Technology, Nazarbayev University, Astana, 010000, Republic of Kazakhstan; \\ 2. Shandong Analysis and Test Center, Shandong Academy of Sciences, 19 keyuan Street, Jinan, 250014, P.R. China; \\ 3. College of Basic Medicine, Wuhan University, Wuhan, 430071, P.R. China; \\ 4. Department of Mechanical Engineering, Vanderbilt University, Nashville, TN, 37203, USA; \\ 5. Department of Biochemistry and Cancer Biology, Meharry Medical College, Nashville, TN, 37201, USA. \\ ${ }^{*}$ Co-first authors \\ $\square$ Corresponding author: yingqiu.xie@nu.edu.kz Dr. Yingqiu Xie, Department of Biology, Nazarbayev University, School of Science and Technology, 53 \\ Kabanbay Batyr Ave., Astana, 010000, Republic of Kazakhstan Tel: +7 7172-70-4686 \\ (1) Ivyspring International Publisher. This is an open access article distributed under the terms of the Creative Commons Attribution (CC BY-NC) license \\ (https:// creativecommons.org/licenses/by-nc/4.0/). See http://ivyspring.com/terms for full terms and conditions.
}

Received: 2016.10.17; Accepted: 2016.12.30; Published: 2017.03.12

\begin{abstract}
Receptor tyrosine kinase EGFR is usually localized on plasma membrane inducing the progression of many cancers including malignancy in children (Bodey et al. In Vivo. 2005, 19:931-41), but it contains a nuclear localization signal (NLS) that mediates EGFR nuclear translocation (Lin et al. Nat Cell Biol. 2001, 3:802-8). In this report, we claim that NLS of EGFR has an old evolutionary origin. In particular, our analysis of protein-protein interaction maps reveals that nuclear EGFR (nEGFR) pathways are different from that of membrane EGFR and EGF is not found in nEGFR network, while androgen receptor (AR) is found, which suggests the evolution of prostate cancer, a well-known AR driven cancer, through changes in androgen- or EGF-dependence. Database analysis shows that nEGFR correlates with the tumor grades especially in prostate cancer patients. Structural prediction analysis indicates that NLS can compromise the differential protein binding to EGFR through stretch linkers with evolutionary mutation from $\mathrm{N}$ to $\mathrm{V}$. In the experiment, elevation of nEGFR but not membrane EGFR was found in castration resistant prostate cancer cells. Finally, systems analysis of NLS and transmembrane domain (TM) suggests that NLS has an old origin while NLS neighboring domain of TM has undergone accelerated evolution. Thus, nEGFR has an old origin resembling the cancer evolution, but TM may interfere NLS driven signaling for natural selection of survival to evade NLS induced aggressive cancers. Our data support NLS is a dynamic inducer of EGFR oncogenesis during evolution for advanced cancers. Our model provides a novel insight into the evolutionary role of NLS in oncogenic kinases in cancers.
\end{abstract}

Key words: NLS, EGFR, prostate cancer.

\section{Introduction}

One of the prominent features of eukaryotic cells is the spatial separation of genetic materials from the translational machinery by the nuclear envelope $(1,2)$. Molecules smaller than $40 \mathrm{kDa}$ can passively diffuse through the nucleus envelope, whereas compounds greater than $40 \mathrm{kDa}$ require an active transport, which is mediated by the signal peptide named as nuclear localization signal or sequence (NLS) (3). NLS guided transport is mediated by assisting proteins such as importins (1). NLS can be classified into two major categories: monopartite and bipartite (4). The monopartite NLS contains only one cluster of basic amino acids, while the bipartite NLS has two clusters of basic amino acids separated by a linker region of $10-12$ residues $(2,5,6)$.

Dysregulation of NLS-mediated transport has 
been found in many cancers (7). For example, dysregulated nuclear transport of NF- $\mathrm{KB}$ is associated with cancers $(2,7,8)$. Recent studies showed that nuclear epidermal growth factor receptor (nEGFR) is essential in cancer (9). EGFR is a receptor tyrosine kinase which is normally localized on the plasma membrane (9). Upon binding to EGF, membrane EGFR dimerizes leading to kinase activation (10) to stimulate several downstream signaling pathways, such as PLC- $\gamma-P K C$, PI-3K-Akt-mTOR, and JAK2-STAT3 (11). These oncogenic pathways are essential in tumorigenesis, cancer progression, survival, resistance to chemotherapy, and metastasis $(11,12)$.

Nuclear localized EGFR has been found in regenerated hepatocytes, benign and cancerous tumors $(9,11,13,14,15)$. nEGFR is induced by chemotherapy and radiation therapy (10), indicating its association with the drug resistance. Sequencing analysis uncovered that EGFR nuclear translocation is mediated by its tripartite NLS that contains three clusters of basic amino acids separated by linker regions at 2-3 residues in length $(9,16)$. The NLS of EGFR has the sequence of amino acids as 645 RRRHIVRKRTLRR 657 (9).

nEGFR plays essential roles in transcriptional regulation, protein phosphorylation, and physical interactions with other molecules $(9,14)$. As a transcriptional regulator, nEGFR can activate the expression of various genes related to chromosome instability, cell proliferation, inflammation, and resistance to cancer drugs that include cyclin D1, iNOS, b-Myb, Aurora-A, cyclooxygenase-2, c-Myc, thymidylate synthase, BCRP, and STAT1 $(9,11,13,14,16,17)$. Interestingly, nEGFR does not contain a DNA-binding domain but can form a complex with other proteins to activate transcription $(11,16)$. The information on nEGFR and membrane EGFR crosstalk with other pathways during evolution is not well analyzed. Studying the protein-protein interaction maps during evolution would reveal the whole networks of nEGFR and membrane EGFR in differential signaling pathways for natural selection.

In clinical studies, enhanced expression of nEGFR has been found in many cancers, such as breast carcinoma (18, 19), ovarian cancer (20), non-small cell lung cancer (21), gallbladder carcinoma (22), and oropharyngeal (23). Recently, nEGFR elevation has been found to promote bone metastasis of prostate cancer through repression of miR-1 thereby elevating miR-1 target of TWIST1, an EMT inducer (25). In clinical samples of prostate cancer, nEGFR levels were inversely correlated with the miR-1 tumor suppressor (25). Xia et al. found nEGFR levels were inversely related to the overall survival of ovarian cancer patients (26). It is worth mentioning that prognostic value of the high levels of nEGFR still remains unclear in many cancers (27). This study aims to investigate the original or evolved form (nuclear or membrane) of EGFR during cancer development and EGFR contributions to the evolution of cancer using systems biology approach.

\section{Materials and Methods}

\section{Nuclear localization signal reference and sequence search}

The NLS sequence in human EGFR, 645 RRRHIVRKRTLRR 657, was used as a reference for the comparison of the NLS (9) in randomly selected species. The sequences of selected species were obtained from GenBank database.

\section{Correlation analysis of nuclear EGFR and cancer progression using database sets}

The PubMed and google scholar databases were used for literature searching for expression of nuclear EGFR in cancers. Several studies were reviewed to find correlations between nuclear EGFR and cancer development (19, 25, 26, 29). Membrane EGFR expression data were used as a control. Pearson correlation was used to measure a linear relationship between expressions of EGFR with cancer stages.

\section{Construction of protein-protein interaction maps and prediction of protein structures}

STRING software was used to construct interaction maps of membrane EGFR and nuclear EGFR respectively (http://version10a.string-db. org/). Protein structures were predicted by inputting sequences to online software (http://raptorx. uchicago.edu/StructurePrediction/) and NLS regions were marked.

\section{Cell culture, treatment and western blot}

LAPC4 cells were cultured in DMEM medium (Life Technologies) with or without androgen depletion treatments by charcoal striped FBS (Life Technologies) for 7 days and were passaged twice. Cells were lysed in buffer provided by the Qproteome Cell Compartment Kit (Qiagen), and cell lysates from nucleus, cytosol, and membrane were subjected to western blot analysis (protocol provided by BioRad) with antibodies of EGFR (Cell signaling), PARP (Cell signaling), and HSP90 (Cell signaling).

\section{Analysis of nuclear localization signal conservation and construction of phylogenetic trees}

Protein BLAST was used to obtain identity percentage of the full-length EGFR protein and NLS 
sequence in the randomly selected species. A cladogram was used to estimate overall organisms' relatedness and conservation of NLS in different species (25). EGFR sequences of all animals for which EGFR sequences were discovered were used for further construction of phylogenetic trees with tools as described in supplementary figure legend.

\section{Results}

\section{EGFR is highly conserved among species closely related to human}

The presence and evolutionary relationships of EGFR among various species were studied. The hypothesis states that the possession of high identity in EGFR protein will indicate that species are closely related to each other during EGFR evolution. To test this, we analyzed 11 randomly selected species using BLAST database. We found the percentage identity in the full-length EGFR relative to human EGFR sequence (Fig.1A). Our analyses showed that EGFR is conserved throughout various species. For example, Pygmy chimpanzee, Horse, Dog, Norway rat and House mouse of EGFR sequence possess 100\% identity with human EGFR (Fig. 1A). Thus, EGFR is conserved in mammalian species closely related to human.

\section{Nuclear localization signal of EGFR is highly conserved among species with the prostate}

We were interested in understanding how EGFR is translocated into the nucleus. By taking the advantage of recent finding about NLS sequence (RRRHIVRKRTLRR) (9), we decided to determine the evolutionary origin of this nuclear event. Therefore, we used the sequence of human EGFR NLS as a reference to compare with previously chosen species. Surprisingly, compared to the full-length EGFR, more organisms have a $100 \%$ identity with human NLS, namely, Pygmy chimpanzee, Horse, Dog, House mouse, Sheep, and Green sea turtle, Chicken (Fig. 1B). By contrast, NLS sequences in Burmese python, Norway rat, Elephant shark and Western clawed frog were less identical to human NLS. We found incidences of different percentages in identity (Fig. 1B). Hence, we focused on the establishment of phylogenetic characterization of selected species.

We next investigated whether there was any correlation between the full length EGFR conservation and the presence of NLS. To estimate the extent of NLS conservation in comparison with full length EGFR sequence, the relative percentage difference for similarity was obtained by a ratio of similarities of NLS vs full length EGFR. Our results demonstrate that Human, Chimpanzee, Norway rat, Horse, Dog,
Western clawed frog, House mouse and Sheep have less than $10 \%$ difference in full length EGFR sequence and NLS sequence (Fig. 1C). Most of these animals have prostate organs (Fig. 1C). Meanwhile, Elephant shark, Burmese python, Green sea turtle, and Chicken account for more than $20 \%$ difference between NLS sequence and full length EGFR sequence (Fig. 1C). Thus, our results suggest that the conservation and evolution of the full length EGFR and NLS are independent events.

\section{Nuclear localization signal of EGFR has an old evolutionary origin}

To determine whether nEGFR is an old or modern evolutionary event, phylogenetic analysis was performed. First we used a cladogram to estimate overall organism's relatedness and conservation of NLS in different species (data not shown) $(25,30)$. Human has a common ancestor with elephant Shark, Jaws, as well as Western Clawed frog and Four-Legged (31). NLS similarities of human with these species above are $84.61 \%$ and $76.92 \%$, respectively. Furthermore, Green sea turtle and Burmese Python showing NLS similarities of $100 \%$ and $92.31 \%$, respectively, were found to come from Amniotic Egg ancestor. From the previously mentioned examples, we can see that some species from older periods, such as Elephant Shark, show higher identity percentages of NLS of EGFR. Thus, our analysis suggests that NLS of EGFR has an old evolutionary origin.

\section{The mutations of nuclear localization signal of EGFR remain the similar character of amino acids}

To further examine the NLS of EGFR sequence in evolution, amino acid analysis of NLS sequences was performed from randomly selected species. Our analysis reveals that human NLS of EGFR (RRRHIVRKRTLRR) has three upstream stretches of basic amino acids (RRR, RKR and RR, here $R$ is arginine, $\mathrm{K}$ is lysine, which are known to be positive and basic polar amino acids). In addition, threonine and leucine amino acids in the RRRHIVRKRTLRR sequence are conserved among all species (32). The combination of isoleucine and valine amino acids is present in the majority of species. Moreover, some specific changes of amino acids are present in Western clawed frog, Burmese python, and Norway rat. Specifically, the Western clawed frog has SR amino acids instead of RH in human; Burmese python has R amino acid instead of $\mathrm{H}$; Norway rat has QL instead of HI. In the most cases, amino acids are evolved in or changed to the same character for NLS. For example, in the Western clawed frog and Burmese python 
histidine was substituted to arginine, which is also basic amino acid. The analysis suggests that NLS is conserved in the component of amino acids to allow the conservation of nuclear EGFR. We next asked whether the differences in NLS among species corresponded with their tendency to acquire cancer. To clarify, whale and rat have significantly low rates of cancer, so possibly they have evolved evolutionary mechanisms that protect their cells from becoming cancerous. It is known that Dog has a similar tendency to develop cancer as Human, and Green sea turtle has cancer too (33). As shown in Figure 2, Elephant shark and Norway rat have a low identity with Human NLS, which correlates to the low chance of developing cancer.

\section{Nuclear localization signal of EGFR reflects the macro-environmental factors for prostate cancer in evolution}

To further examine the role of NLS of EGFR in the development of cancer, we investigated animals' tendency to develop prostate cancer. It is known that only mammals have the prostate organ. As shown in Figure 1 and 3, NLS of EGFR reflects the prostate organ formation during evolution. Although there are variations in NLS, 100\% conserved NLS are found in majority of species having prostates.

Coffey proposed that among mammals, human and dog develop prostate cancer due to the consumption of meat (34) (Fig.2). It is well known that blood serum contains EGF. Thus, raw meat eater or carnivore animals may have higher chances to evolve cancer because if EGF/EGFR signaling activates cancer through bloody raw meat eating, herbivore animals may have low chances to get cancer (Fig.2). Moreover, consumption of green tea, isoflavones, lycopenes, and cruciferous vegetables can reduce the chances of prostate cancer (35). Plant phenolics, including curcumin, genistein and catechins, are known as potential inhibitors of cancer (36). For example, curcumin inhibits EGFR activity and EGF expression, thus decreases the invasiveness of cancer (37). To conclude, the diet diversity of plant foods and meat most likely has the effect on prostate cancer evolution through the reflection of NLS evolution and cooperation with environmental factors.

B

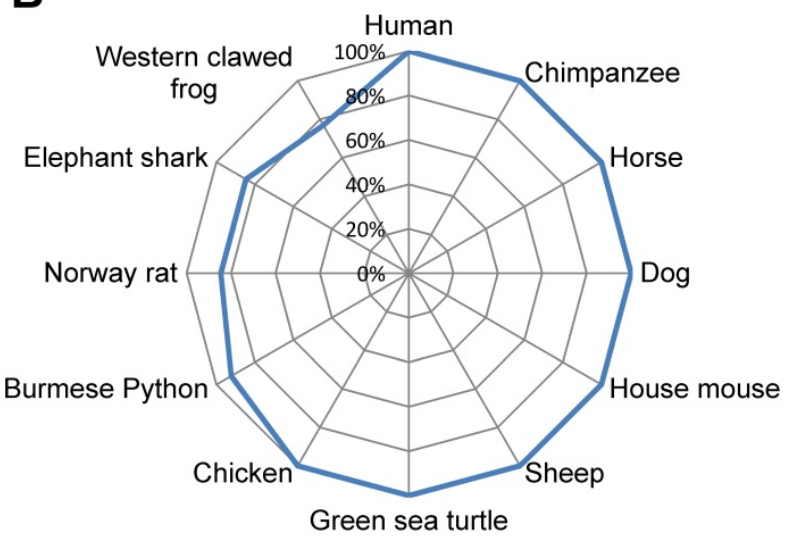

A

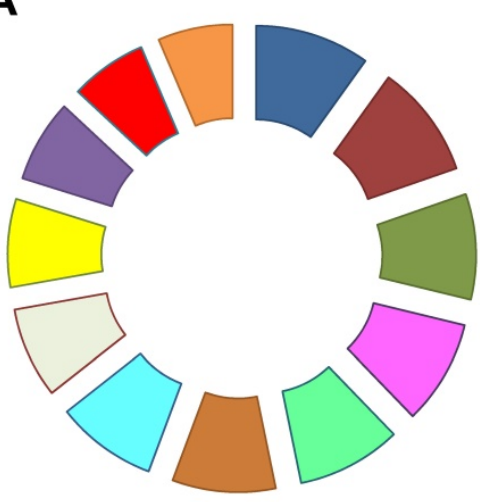

-Human

घChimpanzee

a Horse

$\square$ Dog

$\square$ Norway rat

$\square$ House mouse

$\square$ Sheep

$\square$ Green sea turtle

$\square$ Chicken

-Burmese Python

- Western clawed frog

$\square$ Elephant shark

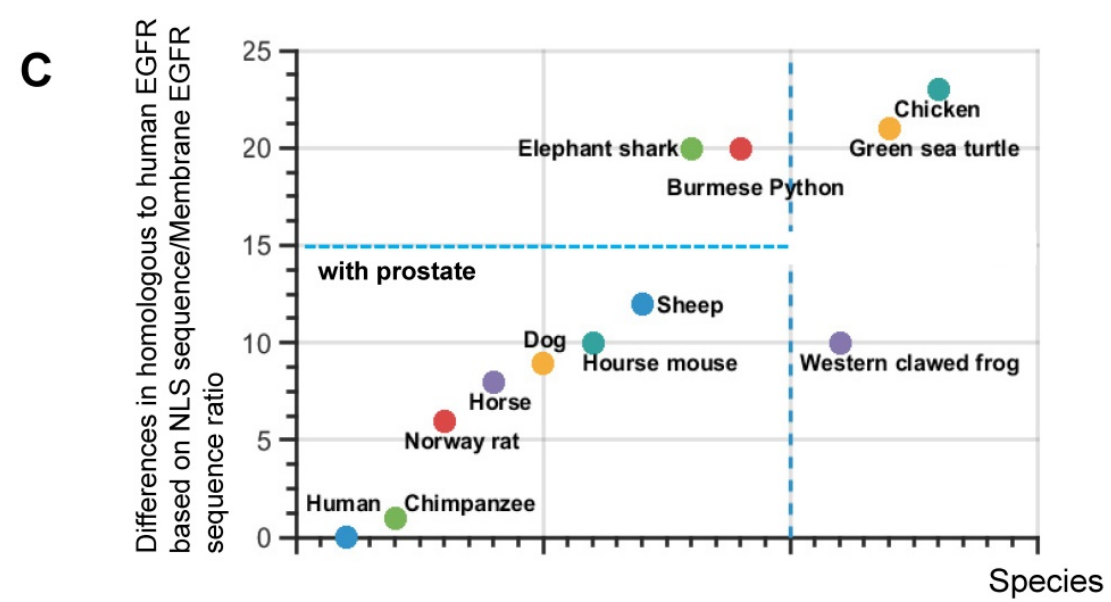

Figure 1. Homologues comparisons of EGFR and NLS of EGFR in different species. A. Similarity of EGFR. B. Similarity of NLS of EGFR. C. Percentage difference in NLS of EGFR and full length EGFR. 


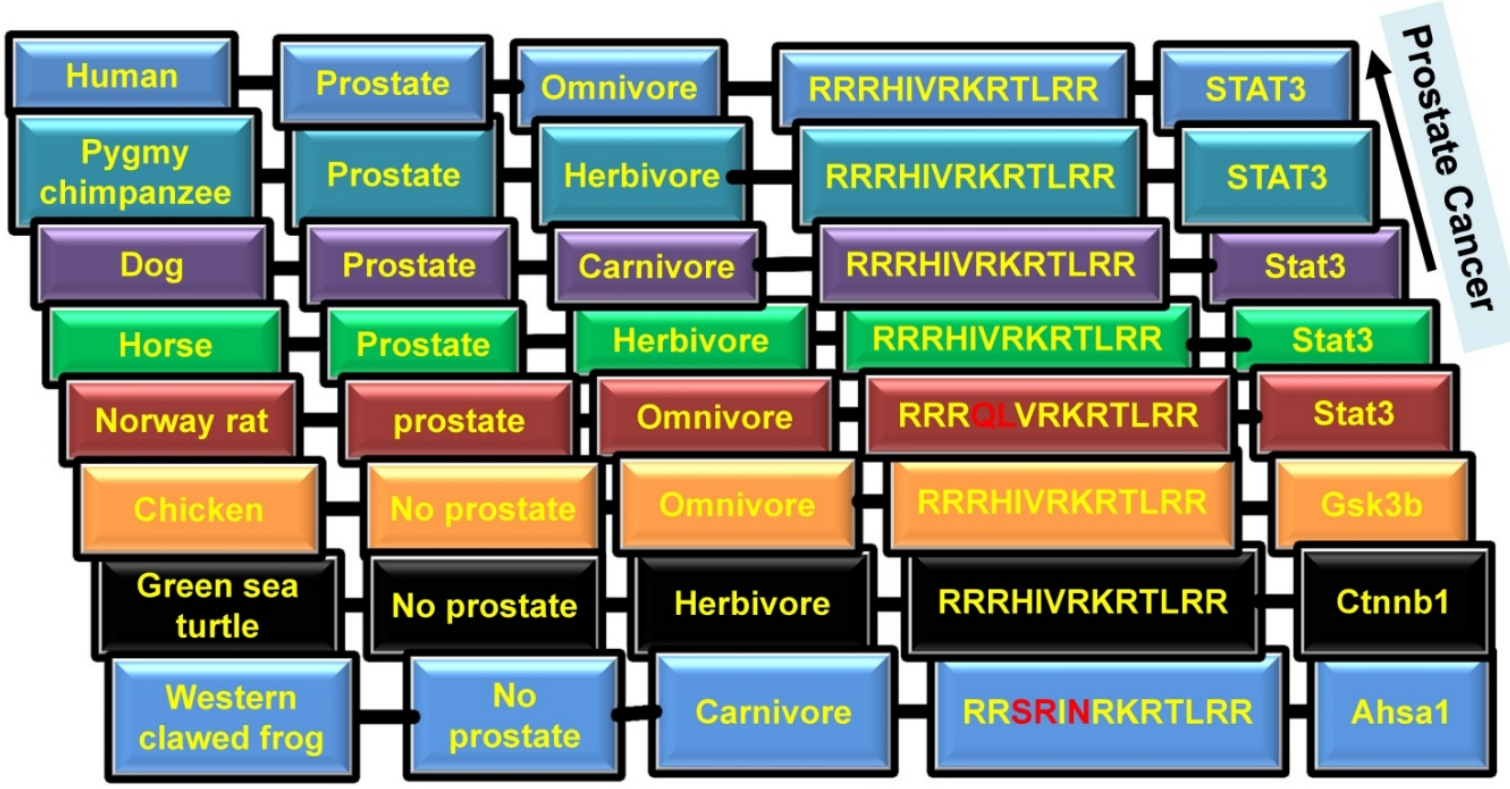

Figure 2. Presence of prostate and types of diet in different species. Columns from left to right: 1. Name of species; 2. Presence of prostate; 3. Type of diet; 4. NLS sequence; 5 . Interaction of EGFR with differential proteins.

\section{Nuclear EGFR correlates to advanced prostate cancer}

To further test our prediction on role of nEGFR in prostate cancer through evolutionary analysis, we searched database to analyze the correlation between nEGFR and prostate cancer development. Clinical studies revealed that nEGFR levels had a correlation with the poor survival of cancer patients. Literatures in PubMed support the correlations between expression of nEGFR with cancer grade $(19,25,26,29)$. Furthermore, there is a direct correlation between nEGFR expression and Gleason score (38) in prostate cancer patients with metastasis (25) (data not shown). Analysis of other three types of cancers also shows the variations in the effect of nEGFR expression on the survival of patients (data not shown). For example, nEGFR expression in breast cancer patients is associated with a poor prognosis, but the expression of nEGFR leads to an inverse correlation with the survival of patients in some cohorts' studies on ovarian cancer $(19,25,26,29)$. In addition, the effect of membrane EGFR on the progression of prostate cancer was investigated using the study of Peraldo-Neia (39). However, the expression of membrane EGFR cannot be used to predict prostate cancer, because there is a poor correlation between the expression of membrane EGFR and cancer progression (39). Moreover, natural selection may play an essential role in nEGFR-mediated evolution of cancer metastasis. Many types of cancers show a negative correlation between nEGFR and cancer stages even nEGFR expressed in all stages of cancers (data not shown). Negative correlation between nEGFR and cancer stages in some types of cancer may suggest that metastatic cancer cells express low levels of nEGFR which may produce the heritable genetic networks of nEGFR to resistant the nEGFR loss due to natural selection. In summary, the expression of nEGFR as a prognostic marker of prostate cancer suggests the organ-specific evolution of cancer driven by nEGFR.

\section{Protein-protein interaction maps suggest the evolutionary networks of nuclear EGFR}

To fully understand the signaling pathways associated with nEGFR in cancer evolution, the protein-protein interaction maps of membrane EGFR and nEGFR were compared. STRING software and previous studies on EGFR pathways were used to construct interaction maps of membrane EGFR and nEGFR (40-44). The results show that common frequent interaction nodes for both nEGFR and membrane EGFR are STAT3, GRB2, BCAR1, and STAT1 (Fig. 3). In addition, the protein interaction maps of membrane EGFR were constructed in different species. We used maps of Zebra Fish and Bos Taurus instead of Elephant shark and Sheep respectively, since they could still give approximate results of EGFR interaction in fish and ruminant domesticated animal. Moreover, the maps for Burmese python and Killer whale were not found in the database of the software. Overall, we observed that EGFR in some species possesses the same interaction proteins as that of human EGFR. For instance, STAT3 protein is presented in all of the 
species which have a close ancestor to human and Pygmy chimpanzee, while Chicken, Green sea turtle, and Western clawed frog do not show interactions between STAT3 and EGFR (string-db.org; supplementary Figure 1). Our data suggest that EGFR interacts with STAT3 in the species closely related to human, which leads to evolutionary transition in the signaling pathway. Figure 2 shows that EGFR in Chicken, Green sea turtle and Western clawed frog do not interact with STAT3 pathways but interact with other proteins such as Gsk3b, Ctnnb1 and Ahsa1. Thus, the data suggest that evolutionary origin of EGFR and its interaction with other signaling pathways can be evolved through cooperation with NLS to induce cancer evolution. Interestingly, as shown in Fig. 3, EGF is not found in nEGFR network nodes while androgen receptor (AR) is found as a node, which suggests the evolution of prostate cancer, a well-known AR driven cancer, through original androgen dependence to androgen independence but EGF dependence to EGF independence with a low androgen reactivation. In summary, protein-protein interaction maps suggest the distinct evolutionary pathways between nEGFR and membrane EGFR even they have common interaction networks. STAT3 and AR may be transition pathways that correlate with the prostate organ formation and accelerate the evolution.

\section{The protein structure predicts the driving force of nuclear localization signal in protein-protein interactions during evolution}

To fully understand how NLS evolution can affect protein-protein interactions for signaling pathway transition, we investigated the structural changes upon NLS evolution. As shown in Figure 4, naturally occurred variations of NLS between frog, chicken, mouse and human can affect the linker region adjacent to the catalytic kinase domain. The stretch structures adjacent to NLS allow NLS to provide the structural diversity for evolution such as rotation. For example, amino acid N in NLS is evolved into $\mathrm{V}$ which loses the open space and allows more twisted structure of linker region to interfere more interactions between NLS with other proteins or peptides. Thus, consistent with the protein-protein interaction maps analysis, structural prediction analysis suggests that NLS may act to compromise the differential protein binding to EGFR during evolution. In summary, our data suggest that NLS is a dynamic driver for EGFR evolution in advanced cancers especially recurrent or therapy resistant cancer. Our model provides novel insights into the evolutionary role of oncogenic NLS in cancer.

\section{Nuclear EGFR}

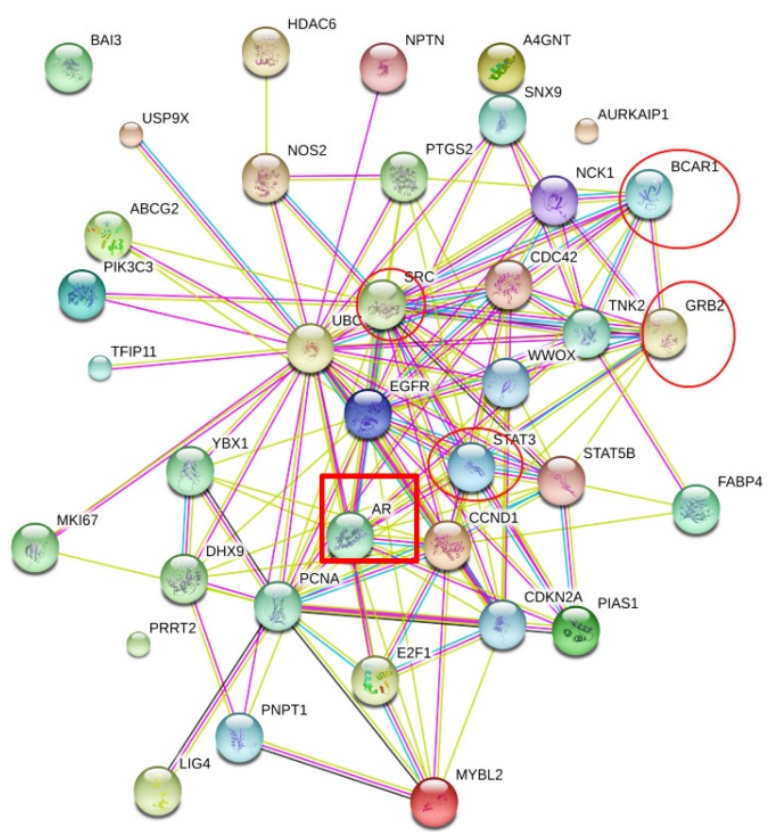

\section{Membrane EGFR}

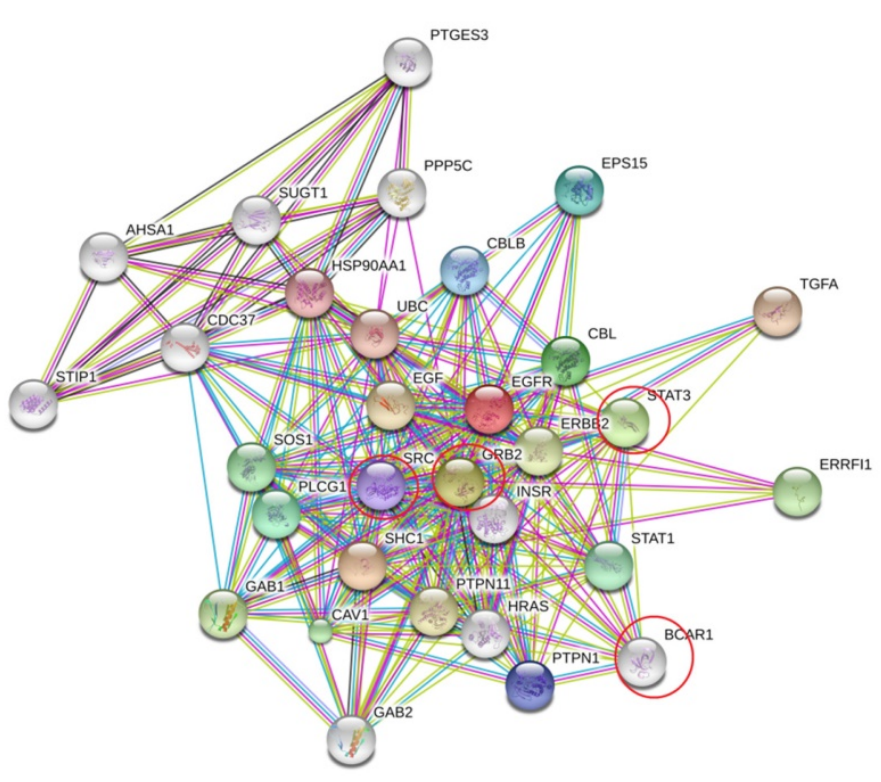

Figure 3. The protein-protein interaction maps of nuclear EGFR and membrane EGFR in Human. The maps were generated by the SRTING 10.0a (http://version10a.string-db.org). 


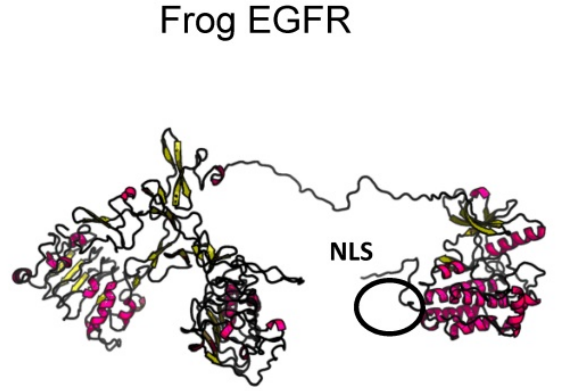

Frog EGFR: NLS

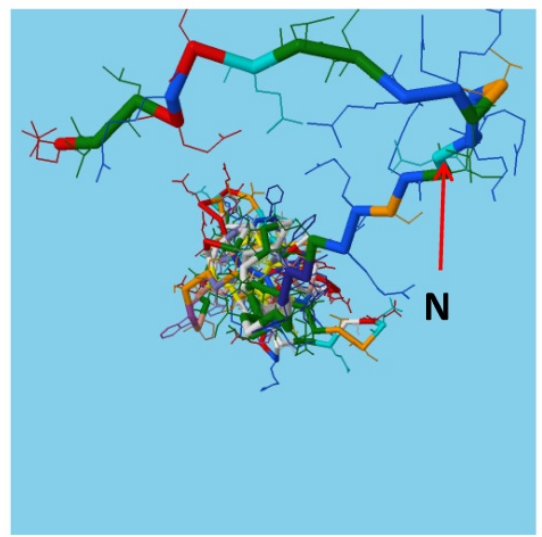

Chicken EGFR

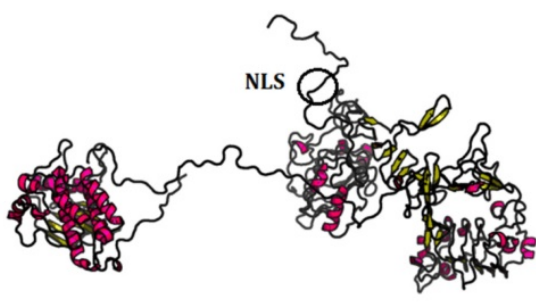

Chicken EGFR: NLS

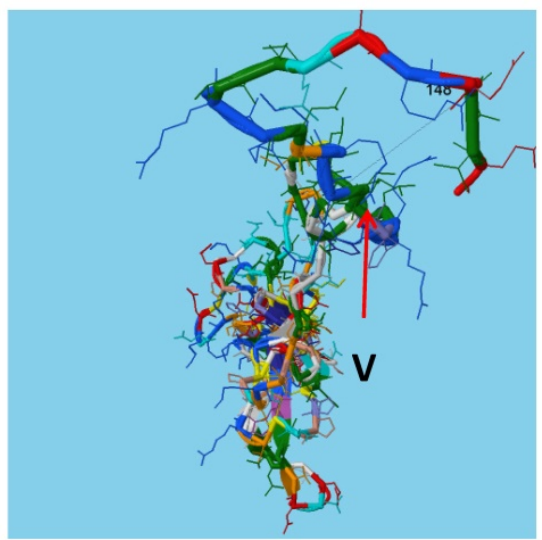

Human EGFR

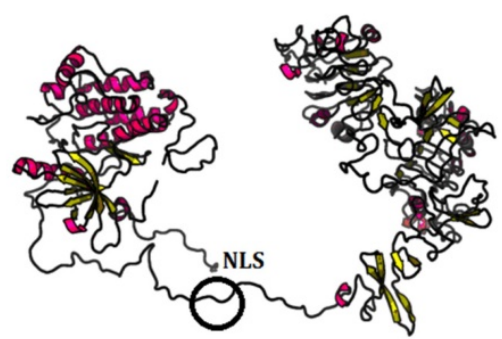

Human EGFR: NLS

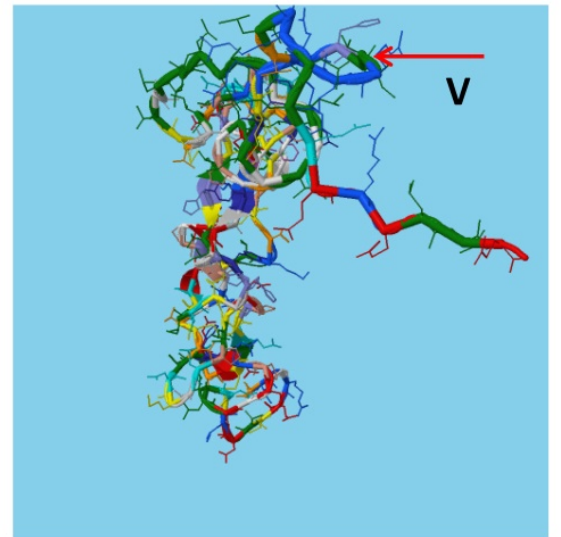

Figure 4. The protein structures predicate the driving force of NLS in protein-protein interactions during evolution. Protein structures were predicated by inputting sequences to software (http://raptorx.uchicago.edu/StructurePrediction/) and NLS regions were marked.

\section{Nuclear EGFR elevation during prostate cancer evolution from androgen sensitive to androgen insensitive}

To further test whether nEGFR indeed plays essential roles in prostate cancer evolution involving in AR pathways, we applied an androgen responsive cell line LAPC4 to monitor the changes of prostate cancer from androgen sensitive to insensitive status. We used charcoal striped FBS (cFBS) to remove androgen and selected cells after 2 passages. Cells survived from androgen depletion were further tested for the expression levels of EGFR in different cellular compartments. As shown in Fig.5, nuclear EGFR but not membrane EGFR was elevated in cells treated with cFBS medium. Cytosolic EGFR was also accumulated, which might be due to an inefficient nuclear translocation in short terms as 7 days during cancer evolution. However, our data support the induction of nuclear EGFR from the transition of androgen-dependence to androgen-independence during prostate cancer evolution.

\section{The systems analysis of nuclear localization signal and transmembrane domain suggest the old origin and accelerated evolution}

To further explore the mechanism of NLS in evolution, we compared the sequences NLS and its nearby domain of TM in 93 organisms. Surprisingly, the systems analysis showed that NLS is much more conserved than TM domain (Supplementary Fig. 2). As EGFR predominately localizes at plasma membrane, TM domain should be conserved. However, we found TM has undergone accelerated evolution in ancestors. Polygenetic trees showed that TM has more branches than NLS. Thus systems analysis further confirms that NLS has an older origin than TM. In summary, our data suggest that nEGFR has an old origin resembling the cancer evolution. Modern TM may prohibit NLS driven pathways to evade NLS induced aggressive cancers. Natural selection for survival may play an important role to against the cancer induced death. Thus, reactivation of nEGFR may allow the historically aggressive cancer to appear.

\section{Discussion}

The review of recent studies shows that tyrosine kinases such as EGFR can localize into the nucleus due to its NLS (9). Thus, it is important to analyze NLS in different organisms to understand its role in the mechanism of nEGFR and the evolution of cancer. The significant similarity in the putative NLS sequences is found among Green sea turtle, Horse, Sheep, Dog, Human, Chimpanzee, Chicken, and 
House mouse. Less similar, but still significant similarity is found among Elephant shark, Western clawed frog, Norway rat and Burmese python (Fig. 1). Although the reduced similarity in amino acid sequence of NLS among species, they possess a similar structure. For example, all organisms possess three stretches of basic amino acids and two sets of nonpolar amino acids ( $\mathrm{I}, \mathrm{V}$ and $\mathrm{L})$. The results suggest that EGFR protein retained the ability to translocate into the nucleus despite some changes of NLS in amino acid. The difference in amino acid sequence might affect the efficiency and rate of nuclear import and mobility of EGFR. It is likely that the degree of nuclear localization is affected by the number of arginine residues in the basic stretch. Figure 2 demonstrates that NLS of EGFR is rich in arginine (R), suggesting that the length of arginine stretch in NLS sequence might affect the internalization of EGFR into nucleus. It can be seen that various organisms have different numbers of arginine. For example, Burmese python's NLS sequence is the richest in arginine residues, whereas Western clawed frog has the least number of arginine in its NLS sequence. The effect of arginine on nuclear localization can be explained by ionic interactions of positively charged arginine with the negatively charged membrane phosphate. In addition, arginine might bind to the extranuclear components such as importin, which results in the nuclear import of EGFR.

To understand how the changes in a single amino acid can affect the nuclear events of EGFR, it will be worth to look at the phylogenetic origin of the analyzed species in relation to Human. Some species showed higher percentage of similarity possessing ancestry with Amniotic Egg from older period being
Carboniferous era 359 Mya (million years ago). Whereas, species having ancestor from Silurian period 443 Mya and Devonian period about 416 Mya showed lower similarity percentages of NLS of EGFR with human. Therefore, it was predicted that NLS of EGFR has an old evolutionary origin based on the results. The finding of percentage differences of NLS and full length EGFR sequence is consistent with the evolutionary species (Fig. 1C). In particular, Human, Pygmy chimpanzee, Dog, Horse, Sheep, Norway rat and House mouse are closely related and have a common ancestor, which is also seen from the percentage difference.

To investigate the development of cancer, it is important to examine animals that have a tendency to develop prostate cancer. In the last 10,000-15,000 years human and dog have significantly changed their diets. That time was a turnover in the diet of Human from plant-feeding to meat-eating, which possibly affects their tendency to develop prostate cancer. Several studies were conducted to further investigate what type of food causes prostate cancer (45). The studies of Kristal et al. figured out that high-grade prostate cancer was correlated with the consumption of polyunsaturated fats, n-6 fatty acids, vitamins D and $\mathrm{E}$, and selenium, whereas nutrition with calcium decreases the chances to develop prostate cancer (46). Another research highlighted that intake of plant food can prevent prostate cancer (35). Moreover, consumption of high total fat, meat, and a multivitamin may increase the incidence of prostate cancer (47). It can be concluded that prostate cancer is a modern event in evolution, which possibly is resulted from dietary changes.
A

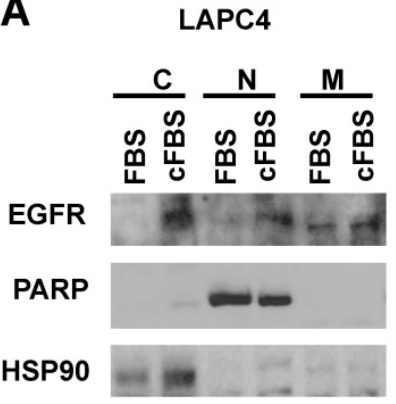

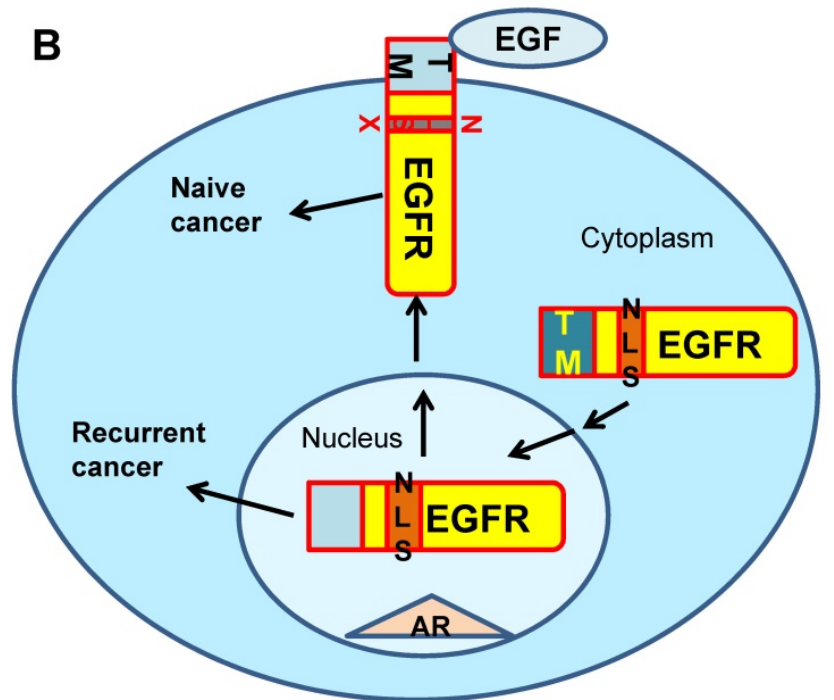

Figure 5. Nuclear EGFR elevation during prostate cancer evolution from androgen sensitive to androgen insensitive. A. LAPC4 prostate cancer cells were androgen deprived for 7 days and expression levels of EGFR in cellular compartment were analyzed by western blot. C: cytosol; N: nucleus; M: membrane. B. Model of EGFR in broad cancer evolution by NLS reactivation through EGF independent pathways. 
Since EGFR could promote tumor progression from various interactions involving signaling pathways, we generated the protein-protein interaction maps on EGFR (48). The analysis of protein interaction maps revealed some proteins interact with both forms of EGFRs, such as STAT1, STAT3, GRB2, and BCAR1 $(17,48-50)$. It was found that the interaction of nEGFR with STAT3 results in the transcriptional activation of iNOS (18). Moreover, IGFBP2 has a role in activation of EGFR/STAT3 signaling and facilitating EGFR accumulation in the nucleus (51). Also, nEGFR interaction maps showed the interaction with androgen receptor (AR). Chen and his colleagues suggested that AR in Sertoli cells regulates the double strand breaks repair and chromosomal synapsis of spermatocytes via EGF-EGFR signaling (52). AR is the key factor in the progression and metastasis of prostate cancer. Taplin et al. found that 5 of 10 patients with distant metastases failed to undergo endocrine therapy, and the authors also found that AR mutations influence the metastatic development of prostate cancer (53). The amplified AR in some cases of prostate cancer is mostly not mutated and therefore retained specificity for androgen $(50,54)$. In addition, AR modifications such as phosphorylation may promote metastatic prostate cancer $(55,56)$.

Protein-protein interaction analysis of membrane EGFR in different species showed that STAT3, which can act as a regulator of genes responsible for cell migration and apoptosis, was found in frequent interaction nodes of species closed to Human such as Pygmy chimpanzee, Norway rat, Horse, and Dog. Other species showed other interacting proteins with EGFR. For instance, Chicken and Green sea turtle both had interaction of EGFR with CBLB, which is known as an E3 ubiquitin-protein ligase regulating $\mathrm{T}$ cell activation, and SHC (Src Homology 2 Domain Containing) Transforming Protein 1 involved in different signaling pathways $(57,58)$. STAT3 protein interacts with EGFR in only closely related species to Human, which suggests that Chicken is the evolutionary transition for different signaling pathways. Chicken and Green sea turtle's EGFR interact with other proteins, CBLB and SHC-1, which can be explained by same amino acid sequence in NLS of EGFR.

To conclude, we found that NLS of EGFR is conserved among different species. Phylogenetic tree analysis shows that NLS of EGFR has an old evolutionary origin. The connection between nEGFR and cancer progression was observed from several clinical studies by database search. The effect of nEGFR expression on cancer development varies with cancer types. Protein-protein interaction maps of
nEGFR and membrane EGFR in different species revealed several proteins being involved in same pattern in closely related species. In addition, it will be important to further investigate how the structure of EGFR protein in the different species may cooperate with NLS to induce the dynamic signaling and cancer evolution.

\section{Supplementary Material}

Supplementary figures.

http://www.jcancer.org/v08p0903s1.pdf

\section{Acknowledgements}

This work was supported in part by Kazakhstan-China collaboration grant, China-Kazakhstan collaboration grant (No. CK-07-09) to Yingqiu Xie and Lixia Miao. We would like to thank Charles Sawyers and Brett S. Carver from Memorial Sloan Kettering Cancer Center for providing the LAPC4 cells and technical support. The authors would like to thank the Department of Biology, SST, and Nazarbayev University for the support of inquired learning, flipped classroom and research integrated teaching innovations of Biochemistry I course for undergraduate students. We would like to thank all the Biochemistry I course students of Nazarbayev University due to their support for the publication of assignment of the Biochemistry I course including this paper and other publications.

\section{Competing Interests}

The authors have declared that no competing interest exists.

\section{References}

1. Lange A, McLane L, Mills R, Devine S, Corbett A. Expanding the Definition of the Classical Bipartite Nuclear Localization Signal. Traffic 2010; 11: 311-23.

2. McLane L, Corbett A. Nuclear localization signals and human disease. IUBMB Life 2009; 61: 697-706.

3. Marfori M, Mynott A, Ellis J, Mehdi A, Saunders N, Curmi P, et al. Molecular basis for specificity of nuclear import and prediction of nuclear localization. Biochimica et Biophysica Acta (BBA) - Molecular Cell Research 2011; 1813: 1562-77.

4. Chang C, Couñago R, Williams S, Boden M, Kobe B. The distribution of different classes of nuclear localization signals (NLSs) in diverse organisms and the utilization of the minor NLS-binding site inplantnuclear import factor importin-a. Plant Signaling \& Behavior 2013; 8: e25976.

5. Christie M, Chang CW, Róna G, Smith KM, Stewart AG, Takeda AA, et al. Structural biology and regulation of protein import into the nucleus. Journal of Molecular Biology. 2015; 1-31.

6. Cautain B, Hill R, Pedro N, Link W. Components and regulation of nuclear transport processes. FEBS Journal 2015; 282: 445-62.

7. Hung MC, Link W. Protein localization in disease and therapy. Journal of Cell Science 2011; 124: 3381-92.

8. Kau TR, Way JC, Silver PA. Nuclear transport and cancer: from mechanism to intervention. Nature Reviews Cancer 2004; 4: 106-17.

9. Brand TM, Iida M, Li C, Wheeler DL. The nuclear epidermal growth factor receptor signaling network and its role in cancer. Discovery Medicine 2011; 12: 419-32.

10. Huang WC, Chen YJ, Hung MC. Implication of nuclear EGFR in the development of resistance to anticancer therapies. BioMedicine 2011; 1: 2-10.

11. Han W, Lo HW. Landscape of EGFR signaling network in human cancers: biology and therapeutic response in relation to receptor subcellular locations. Cancer Letters 2012; 318: 124-34. 
12. Wang YN, Yamaguchi H, Hsu JM, Hung MC. Nuclear trafficking of the epidermal growth factor receptor family membrane proteins. Oncogene 2010; 29: 3997-4006.

13. Lo HW. Nuclear mode of the EGFR signaling network: biology, prognostic value, and therapeutic implications. Discovery Medicine 2010; 10: 44-51.

14. Dittmann K, Mayer C, Paasch A, Huber S, Fehrenbacher B, Schaller M, et al. Nuclear EGFR renders cells radio-resistant by binding mRNA species and triggering a metabolic switch to increase lactate production. Radiotherapy and Oncology 2015; 116: 431-7.

15. Pereira NB, do Carmo AC, Diniz MG, Gomez RS, Gomes DA, Gomes CC. Nuclear localization of epidermal growth factor receptor (EGFR) in ameloblastomas. Oncotarget 2015; 6: 9679-85.

16. Lee HH, Wang YN, Hung MC. Non-canonical signaling mode of the epidermal growth factor receptor family. American Journal of Cancer Research 2015; 5: 2944-58.

17. Brand TM, Iida M, Luthar N, Starr MM, Huppert EJ, Wheeler DL. Nuclear EGFR as a molecular target in cancer. Radiotherapy and Oncology 2013; 108: 370-7.

18. Lo H, Hsu S, Ali-Seyed M, Gunduz M, Xia W, Wei Y, et al. Nuclear interaction of EGFR and STAT3 in the activation of the iNOS/NO pathway. Cancer Cell 2005; 7:575-589.

19. Lo H, Xia W, Wei Y, Ali-Seyed M, Huang S, Hung M. Novel prognostic value of nuclear epidermal growth factor receptor in breast cancer. Cancer Research 2005; 65: 338-348.

20. Xia W, Wei Y, Du Y, Liu J, Chang B, Yu Y, et al. Nuclear expression of epidermal growth factor receptor is a novel prognostic value in patients with ovarian cancer. Molecular Carcinogenesis 2009; 48:610-617.

21. Traynor A, Weigel T, Oettel K, Yang D, Zhang C, Kim K, et al. Nuclear EGFR protein expression predicts poor survival in early stage non-small cell lung cancer. Lung Cancer 2013; 81:138-141.

22. Li C, Fang F, Wang J, Tzeng C, Tai H, Wei $\mathrm{Y}$, et al. EGFR nuclear import in gallbladder carcinoma: nuclear phosphorylated EGFR upregulates iNOS expression and confers independent prognostic impact. Annals of Surgical Oncology 2012; 19:443-454

23. Psyrri A, Yu Z, Weinberger P, Sasaki C, Haffty B, Camp R, et al. Quantitative Determination of Nuclear and Cytoplasmic Epidermal Growth Factor Receptor Expression in Oropharyngeal Squamous Cell Cancer by Using Automated Quantitative Analysis. Clinical Cancer Research 2005; 11:5856-5862.

24. Hoshino M, Fukui H, Ono Y, Sekikawa A, Ichikawa K, Tomita S, et al. Nuclear Expression of Phosphorylated EGFR Is Associated with Poor Prognosis of Patients with Esophageal Squamous Cell Carcinoma. Pathobiology 2007; 74:15-21.

25. Chang YS, Chen WY, Yin JJ, Sheppard-Tillman H, Huang J, Liu YN. EGF receptor promotes prostate cancer bone metastasis by downregulating miR-1 and activating TWIST1. Cancer Research 2015; 75: 3077-86.

26. Xia W, Wei Y, Du Y, Liu J, Chang B, Yu YL, et al. Nuclear expression of epidermal growth factor receptor is a novel prognostic value in patients with ovarian cancer. Molecular Carcinogenesis 2009; 48: 610-7.

27. Ahel J, Dordevic G, Markic D, Mozetic V, Spanjol J, Grahovac B, et al. Nuclear EGFR characterize still controlled proliferation retained in better differentiated clear cell RCC. Medical Hypotheses. 2015; 85: 183-5.

28. Fletcher WA. Computational statistics in molecular phylogenetics (Doctoral dissertation, UCL (University College London)).

29. Dekanic A, Dintinjan RD, Budisavljevic I, Pecanic S, Butorac MZ, et al. Strong nuclear EGFR expression in colorectal carcinomas is associated with cyclin-D1 but not with gene EGFR amplification. Diagnostic Pathology 2011; 6: 108-15.

30. [Internet] Evolution.berkeley.edu. Phylogenetic systematics, a.k.a. evolutionary trees. http://evolution.berkeley.edu/evolibrary/article/ phylogenetics_01.

31. [Internet] UCL. Tree of Life. http://abacus.gene.ucl.ac.uk/will/ files/TreeOfLife.pdf.

32. Nelson DL, Cox MM. Lehninger Principles of Biochemistry. New York: W.H. Freeman and Company; 2013.

33. Merlo DF, Rossi L, Pellegrino C, Ceppi M, Cardellino U, Capurro C, et al. Cancer incidence in pet dogs: findings of the Animal Tumor Registry of Genoa, Italy. Journal of Veterinary Internal Medicine. 2008; 22: 976-84.

34. Coffey DS. Similarities of prostate and breast cancer: evolution, diet, and estrogens. Urology. 2001; 57: 31-8.

35. Hori S, Butler E, McLoughlin J. Prostate cancer and diet: food for thought? BJU International. 2011; 107: 1348-59.

36. Wahle KW, Brown I, Rotondo D, Heys SD. Plant phenolics in the prevention and treatment of cancer. Springer US: InBio-Farms for Nutraceuticals. 2010:36-51.

37. Kaneuchi M, Sasaki M, Tanaka Y, Yamamoto R, Sakuragi N, Dahiya R. Resveratrol suppresses growth of Ishikawa cells through down-regulation of EGF. International Journal of Oncology. 2003; 23: 1167-72.

38. Kweldam CF, Wildhagen MF, Steyerberg EW, Bangma $\mathrm{CH}$, van der Kwast $\mathrm{TH}$, van Leenders GJ. Cribriform growth is highly predictive for postoperative metastasis and disease-specific death in Gleason score 7 prostate cancer. Modern Pathology. 2015; 28: 457-64

39. Peraldo-Neia C, Migliardi G, Mello-Grand M, Montemurro F, Segir R, Pignochino Y, Cavalloni G, Torchio B, Mosso L, Chiorino G, Aglietta M. Epidermal Growth Factor Receptor (EGFR) mutation analysis, gene expression profiling and EGFR protein expression in primary prostate cancer. BMC cancer. 2011; 11: 1-12

40. Lo HW, Hung MC. Nuclear EGFR signalling network in cancers: linking EGFR pathway to cell cycle progression, nitric oxide pathway and patient survival. British Journal of Cancer 2006; 94: 184-8.

41. Roßner F, Gieseler C, Morkel M, Royer HD, Rivera M, Bläker H, et al. Uncoupling of EGFR-RAS signaling and nuclear localization of YBX1 in colorectal cancer. Oncogenesis. 2016; 5: e187.

42. Wang Z, Hu P, Tang F, Xie C. HDAC6-mediated EGFR stabilization and activation restrict cell response to sorafenib in non-small cell lung cancer cells. Medical Oncology. 2016; 33: 1-9.

43. Savio MG, Wollscheid N, Cavallaro E, Algisi V, Di Fiore PP, Sigismund S, et al. USP9X Controls EGFR Fate by Deubiquitinating the Endocytic Adaptor Eps15. Current Biology 2016; 26: 173-83.

44. Dayde D, Guerard M, Perron P, Hatat AS, Barrial C, Eymin B, et al. Nuclear trafficking of EGFR by Vps34 represses Arf expression to promote lung tumor cell survival. Oncogene. 2015 1-9.

45. Masko EM, Allott EH, Freedland SJ. The relationship between nutrition and prostate cancer: is more always better? European Urology. 2013; 63: 810-20.

46. Kristal AR, Arnold KB, Neuhouser ML, Goodman P, Platz EA, Albanes D, Thompson IM. Diet, supplement use, and prostate cancer risk: results from the prostate cancer prevention trial. American Journal of Epidemiology. 2010; 172: 566-77.

47. Davies NJ, Batehup L, Thomas R. The role of diet and physical activity in breast, colorectal, and prostate cancer survivorship: a review of the literature. British Journal of Cancer. 2011; 105: 52-73.

48. Tomas A, Futter CE, Eden ER. EGF receptor trafficking: consequences for signaling and cancer. Trends in Cell Biology. 2014; 24: 26-34.

49. Lin SY, Makino K, Xia W, Matin A, Wen Y, Kwong KY, et al. Nuclear localization of EGF receptor and its potential new role as a transcription factor. Nature Cell Biology. 2001; 3: 802-8.

50. Tan X, Lambert PF, Rapraeger AC, Anderson RA. Stress-Induced EGFR Trafficking: Mechanisms, Functions, and Therapeutic Implications. Trends in Cell Biology 2016; 26: 352-66.

51. Chua CY, Liu Y, Granberg KJ, Hu L, Haapasalo H, Annala MJ, et al. IGFBP2 potentiates nuclear EGFR-STAT3 signaling. Oncogene. 2015; 35: 738-47.

52. Chen SR, Hao XX, Zhang Y, Deng SL, Wang ZP, Wang YQ, et al. Androgen receptor in Sertoli cells regulates DNA double-strand break repair and chromosomal synapsis of spermatocytes partially through intercellular EGF-EGFR signaling. Oncotarget 2016; 7: 18722-35.

53. Taplin ME, Bubley GJ, Shuster TD, Frantz ME, Spooner AE, Ogata GK, et al. Mutation of the androgen-receptor gene in metastatic androgen-independent prostate cancer. New England Journal of Medicine 1995; 332: 1393-8.

54. Koivisto P, Kononen J, Palmberg C, Tammela T, Hyytinen E, Isola J, et al. Androgen receptor gene amplification: a possible molecular mechanism for androgen deprivation therapy failure in prostate cancer. Cancer Research 1997; 57: 314-9.

55. Guo Z, Dai B, Jiang T, Xu K, Xie Y, Kim O, et al. Regulation of androgen receptor activity by tyrosine phosphorylation. Cancer Cell. 2006;10:309-19.

56. Xie Y, Lu W, Liu S, Yang Q, Carver BS, Li E, et al. Crosstalk between nuclear MET and SOX9/ $\beta$-catenin correlates with castration-resistant prostate cancer. Molecular Endocrinology 2014; 28:1629-39.

57. [Internet] Uniprot. CBLB - E3 ubiquitin-protein ligase CBL-B - Homo sapiens (Human) - CBLB gene \& protein. http://www.uniprot.org/uniprot/Q13191.

58. [Internet] Uniprot SHC1 - SHC-transforming protein 1 - Homo sapiens (Human) - SHC1 gene \& protein. http://www.uniprot.org/uniprot/P29353.

59. UniProt Consortium. UniProt: a hub for protein information. Nucleic acids research. 2014 Oct 27:gku989.

60. Yamada KD, Tomii K, Katoh K. Application of the MAFFT sequence alignment program to large data-reexamination of the usefulness of chained guide trees. Bioinformatics. 2016 Jul 4. pii: btw412.

61. Waterhouse AM, Procter JB, Martin DMA, Clamp M, Barton GJ. Jalview Version 2-a multiple sequence alignment editor and analysis workbench. Bioinformatics 2009; 25: 1189-1191. 